\title{
Geriatric Care Outreach Models for the World
}

\author{
Rajesh Chauhan* \\ Family Healthcare Centre, India
}

Submission: March 31, 2017; Published: April 05, 2017

*Corresponding author: Rajesh Chauhan, Family Healthcare Centre, Avas Vikas Colony Sikandra, Agra 282007, India, Tel: +919760021192;

E-mail: drrajeshchauhan@gmail.com

\section{Editorial}

It is a good sign that globally the geriatric population is increasing by the day, and with it the longevity is also increasing. This is bringing up cheers, but may pose certain challenges about provision of geriatric care and management if long term planning and appropriate actions are not taken while we still have the time. The longevity in South Korea is expected to reach 90 years by 2030, and India expects the longevity to be raised by better health and support to 67 years [1]. While developed countries with better resources and comparatively low absolute numbers will have better chance of taking care of their aged, and their resources will not be overwhelmed by rising demands, unlike what should be taken as a sign of warning for already overwhelmed meager and unevenly spread health resources in developing countries. Wars, natural and man-made disasters, scarcity of health services and trained staff to understand and deal with geriatric health issues will be another set of challenges that will complicate the issues.

Specialist care is sparse, costly, and getting costlier by the day. Furthermore, for every organ and system we have a separate specialist. Everyone can't afford it in terms of both time and money, and certainly every different specialist it is not available everywhere at all times. Currently, to fill in the gaps between healthcare demands of the elderly, besides trained geriatricians, we have other healthcare workers and even some quacks filling in the gap. Most of the developing countries are fighting trying to overcome with scarcities of various kinds. There could be a huge gap between the demand and availability of well trained geriatricians as well. At many places we may have quacks filling in the gap. Simply put, there will be a need of greater numbers of doctors trained in Geriatrics who have in-depth knowledge about various illnesses, morbidities, and other special problems afflicting the aged, including frailty, generation gap, economic, and caregiver issues, etc.

When it would come to prioritization, it is a common knowledge that the aged are getting a delayed priority as compared to the sickness and health issues of children and the able bodied youth and the middle aged. They are at the tail end of priority list, and this would need a change and a permanent make over as longevity increases, with lots of additional inputs and facilities for the aged. For the well endowed, health insured, and for those from well developed nations of the world where standard healthcare facilities are being provided, there will be no problem and no problems of unmet healthcare needs as well. The problems will possibly be seen in poor, developing nations with meager healthcare resources, which may be far outstretched at some places thereby leaving many gaps as well.

Enough for extrapolating for the geriatric age group, as what is really required is starting new researches for all the health problems of the aged, and looking afresh without having to look through stained glasses. It is a time for doing away with the necessity to extrapolate onto them of what's being done for the middle aged and younger age groups in the absence of deliberate new research for the health issues involving the elderly.

Everything that is good and perfect for other age groups may not be so appropriate for geriatric age group as well. In a recent published article, it has been found that total knee replacement (TKR) has minimal benefits. TKR may not be the best option [2]. Likewise, there might be many remediation modalities that we may be following for want of any better or safe options. We have also failed in a way by not picking up the small leads, perhaps due to overlook, or disbelief, or from deficiency in research, etc. Well, if it is because of any deficiency in research, wider and well organized researches could be carried out. Maybe there are already very simple and innovative techniques that could help the aged, and especially the poor who can ill afford the established practices. For the benefit of geriatric population, our thrust should be for management of multi-morbidities, and rejuvenation, preferably with non-surgical and non-invasive painless techniques. Perhaps it's time to move on to individually tailored and focused treatment, especially for the aged [3].

We are also in an era of the internet, where tele-health, eHealth and $m$ Health etc, can be incorporated for the management and 
care of the aged. It will help all those elderly patients who are either immobile or have decreased mobility for various reasons, and also for those who dislike or avoid stepping out of their comfort zones. A dedicated pool of geriatric specialists can be employed to help through eHealth system. Add to this the doorto-door help by a health team, as has already been deliberated earlier [4]. This door to door visit by a compact health team can be supplemented by eHealth. It would help re-organize the healthcare delivery and access in shortest possible time. Just a change of mindset is all that is required. With governmental or NGOs intervention, small eHealth, mHealth, and tele-health hubs can be established at convenient locations across the world to cater for worldwide elderly patients. Quality must be maintained for such hubs to be successful.

'Mobile eHealth Centers' could be based on a platform of self-contained mobile vans/ boats/ etc. They can be tasked with visiting each locality under their charge once a week, check and treat common ailments, provide health counseling, run routine tests and collect samples to be dispatched to reference labs, do X-rays if needed, promote video-conferencing with senior specialists who are readily available at "Health District Centers" (HDCs) and also at tertiary care and teaching hospitals for this help, and finally provide a refill of medicines for seven days until their next visit. This can be achieved by a complement of a well trained team comprising of

A. A doctor who is an expert or specialist in Geriatrics

B. Health Counselor

C. Male and female nurses

D. Lab Technician

E. Physiotherapist

F. Dentist

G. One expert in health informatics, who can help in telemedicine and can handle collection and dissemination of medical data

H. Pharmacist

I. A physically fit and well trained driver who also knows is also trained in CPR and provisioning of first aid.
If this model is followed, besides cost reductions and prevention of unnecessary wastage of resources and efforts, many unwanted hospital and clinic visits can be prevented, while home based and self-care can be promoted within reasonable limits. By extending screening and preventive services, health status can be improved markedly. Generation of clinical alerts \& reminders would be an added feature as would be a paperless atmosphere. All this can help in better planning, budget allocation and utilization, easy monitoring and audits of various types, prevention of wastage and of unwarranted or futile interventions, better continuity and follow up, and ushering in of some degree of accountability as well. That said, it shall always be prudent to take small steps initially, and build from there based on whatever has been actualized and also from the experiences generated.

It may seem somewhat out of place out here, but it is a fact that getting treated and continuing with treatment is costly and at times beyond reach of many who can ill afford it. There may be those who are not insured, poor and for those who are not well endowed, or where there is no adequate governmental support. To bring down the costs of medicines and prevent wastage, wouldn't it therefore be fair for the world's scientists to come together and find effective ways for extending the shelf life of all drugs and disposables in some cost effective manner? Surely the extended shelf-lives of medicines and disposables can be a boon in the long run, and can bring their costs down drastically.

\section{References}

1. Kontis V, Bennett JE, Mathers CD, Li G, Foreman K, et al. (2017) Future life expectancy in 35 industrialised countries: projections with a Bayesian model ensemble. Lancet 389(10076): 1323-1335.

2. Ferket BS, Feldman Z, Zhou J, Oei EH, Bierma Zeinstra SM, Mazumdar M (2017) Impact of total knee replacement practice: cost effectiveness analysis of data from the Osteoarthritis Initiative. BMJ 356: j1131.

3. Chauhan R, Parihar AKS, Chauhan S (2016) Perhaps its Time to Move on to Individually Tailored and Focused Treatment in the Aged Population. J Gerontol Geriatr Res S5: 001.

4. Chauhan R, Parihar AKS, Chauhan S (2013) A new model for establishing effective and sustainable rural healthcare in India, as well as other developing countries around the world. BMJ.

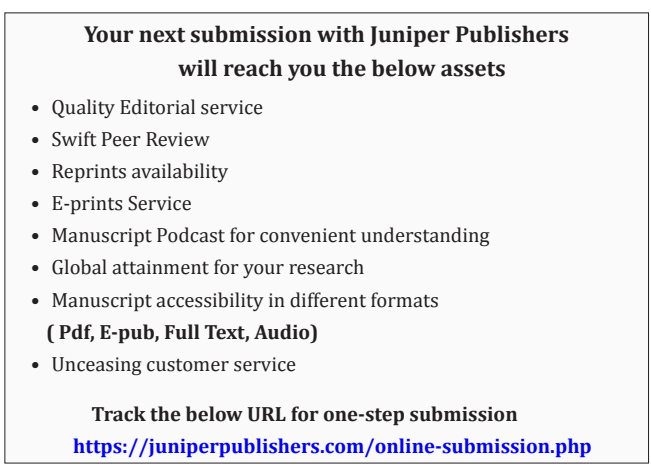

